\title{
Objetos de Aprendizagem Moveis - Uma análise de requisitos funcionais para auxiliar os desenvolvedores.
}

\author{
João Roberto Ursino da Cruz ${ }^{1,2}$, Wellington Barbosa Rodrigues ${ }^{2}$ \\ 1 Mestrado em Ciência da Computação - Faculdade Campo Limpo Paulista \\ (FACCAMP) \\ Rua Guatemala, 167 - Campo Limpo Paulista - SP - 13231-230 - Brasil \\ ${ }^{2}$ Departamento de Tecnologia e Comunicação - Faculdade Sumaré \\ Rua Capote Valente, 1121 - São Paulo - SP - 05409-003 - Brasil \\ joaocruz@cc.faccamp.br, wellington.rodrigues@sumare.edu.br
}

\begin{abstract}
The study on mobile learning has been growing very quickly and developers of learning objects need to adapt to this new reality. One of the main differences of this new reality is that the decision of when to use the LOM belongs to the students and no longer to the educational center. This article reports a study with 147 students and teachers about your preferences in relation to some of the functional requirements for the development of an LOM more enjoyable.
\end{abstract}

Resumo. O estudo sobre mobile learning vem crescendo muito rápido, e os desenvolvedores de objetos de aprendizagem precisam se adaptar a essa nova realidade. Uma das principais diferenças dessa nova realidade é que a decisão de quando usar o OAM pertence ao aluno, e não mais dos centros educacionais. Esse artigo relata um estudo com 147 alunos e professores sobre suas preferencias em relação a alguns dos requisitos funcionais para o desenvolvimento de um OAM mais agradável.

\section{INTRODUÇÃO}

Desde que Mark Weiser em 1980 definiu o termo "Computação Ubíqua”, o mundo passou a ver com outros olhos para os dispositivos móveis e suas aplicações, o próprio Weiser (1993) passa a chamar esse olhar como "terceira onda" da computação. E a computação Ubíqua em muito pouco tempo atingiu as escolas com o mobile learning e os objetos de aprendizagem móveis.

Segundo a PNAD $^{1}$ realizada no ano de 2014, 93,4\% dos estudantes da rede privada de ensino e $68 \%$ dos estudantes da rede pública possuem pelo menos um dispositivo móvel o que totalizaria mais de $74,3 \%$ de estudantes com potencial para o aprendizado através de Objetos de Aprendizagem Móveis (OAM). Um número tão grande de dispositivos moveis presentes dentro das escolas, faculdades e centros formadores, fez crescer ainda mais os estudos sobre o mobile learning e os OAMs, o que aproximou professores, desenvolvedores e pesquisadores no esforço para a criação de OAMs mais atrativos para os alunos

A importância do mobile learning para a formação do ser humano ganhou uma

\footnotetext{
${ }^{1}$ PNAD - Pesquisa Nacional por Amostra de Domicílios é realizada pelo IBGE.
} 
V Congresso Brasileiro de Informática na Educação (CBIE 2016)

Anais do XXVII Simpósio Brasileiro de Informática na Educação (SBIE 2016)

importância tão grande, que West e Vosloo (2012) afirmam em que "Como as tecnologias móveis continuam a crescer em poder e funcionalidade, sua utilidade como ferramentas educacionais deverá expandir muito e, com isso, sua centralidade na educação formal, bem como informal. Por estas razões, a UNESCO ${ }^{2}$ acredita que a aprendizagem móvel merece o cuidado".

Este artigo relata um estudo realizado com professores e alunos dos diversos níveis educacionais (do Ensino Fundamental ao Doutorado) visando conhecer melhor suas realidades e opiniões sobre os requisitos necessários para o desenvolvimento de um OAM.

$\mathrm{Na}$ Seção 2 são apresentados os objetivos deste estudo, suas fundamentações, o protocolo e a execução da pesquisa. Na Seção 3 são apresentados os resultados obtidos, e finalmente na Seção 4 são feitas algumas considerações sobre esses resultados.

\section{OBJETIVOS E FUNDAMENTAÇÃO PARA DEFINIÇÃO DE REQUISITOS DE UM OAM}

Muitos dos requisitos necessários para o desenvolvimento de um OAM são herdados dos demais Objetos de Aprendizado Computacionais, mas conforme é possível ler em Cruz, Ramos e Rodrigues (2015) "os profissionais da educação devem buscar novas formas de atrair a atenção e interesse dos alunos visando uma melhor prática de ensino (..)", ou seja, um OAM deve ser agradável e atrair o aluno, pois seu uso deverá acontecer não apenas na sala de aula, mas em vários ambientes.

Georgieva (2009) em seu artigo faz uma análise comparativa entre diversos tipos de aplicativos mobile learning, e enfatiza que não existe um padrão único ao falarmos de OAMs. Essa diversidade de tipos de OAM são em parte reflexo de diversidade de formas pedagógicas de se apresentar conteúdo diferentes para alunos com realidades diferentes. Sempre que possível os desenvolvedores e professores deverão conhecer essas realidades e os gostos dos alunos antes de definir qual será o tipo de OAM

Moreira e Conforto (2011) dividem as funcionalidades de um OA em dois tipos as funcionalidades do sistema, que atendem a plataforma $\mathrm{e} o$ ambiente $\mathrm{e}$ as funcionalidades didáticas, que atendem a finalidade do ensino pelo uso do OA. Ainda, segundo Yen e Lee (2011) afirmam que sempre que possível deverão ser desenvolvidos funcionalidades que promovam a interatividade entre o aluno, o ambiente e o professor.

Sharp et al. (2003) também ressaltam que o desenvolvedor de um OAM deve possuir um plano para readequação dos conteúdos originalmente projetados para serem usados em outras plataformas, pois é esperado que essa aplicação não apenas apresente os conteúdos a serem estudados, mas use os demais recursos da plataforma para estimular o aluno a buscar um maior conhecimento fazendo com que o aplicativo e esses recursos interajam em uma linguagem dinâmica.

Moreira e Conforto (2011) também enfatizam que os OA devem possuir ferramentas de acessibilidade em conformidade com a Política Nacional de Educação Especial (MEC/SEESP 2007).

A partir das afirmações dos autores temos como objetivo ouvir professores e alunos sobre requisitos como: quais os tipos de aplicações prediletas pelos alunos, a

\footnotetext{
${ }^{2}$ UNESCO - United Nations Educational, Scientific and Cultural Organization
} 
V Congresso Brasileiro de Informática na Educação (CBIE 2016)

Anais do XXVII Simpósio Brasileiro de Informática na Educação (SBIE 2016)

linguagem a ser usada nas interfaces, interatividade, recursos multimídias, e acessibilidade de um OAM.

\section{Protocolo}

Para realização da pesquisa foram definidos dois grupos de voluntários. O primeiro grupo composto por professores, independentemente do nível de ensino ou instituições no qual atuam ou atuaram, e o segundo composto por alunos matriculados em instituições de ensino independentemente do nível de ensino.

Foram elaborados dois modelos de questionários, um para cada grupo, ambos possuíam 4 seções visando identificar o perfil dos entrevistados, suas habilidades e conhecimento no uso de dispositivos móveis, suas opiniões sobre algumas funcionalidades oferecidas nos OAMs e a opinião dos mesmos sobre o uso de dispositivos móveis em ambientes de ensino. As contribuições deveriam ser espontâneas e antes de responder ao questionário os voluntários deveriam ler um TCLE (Termo de Consentimento Livre e Esclarecido) que esclarecia quais as finalidades da pesquisa e concordar com a mesma. Foram excluídos da pesquisa qualquer voluntário que não se enquadrasse no perfil estabelecido, ou não concordasse com o TCLE.

\section{Execução}

O questionário foi construído usando a ferramenta Google Forms, e disponibilizado pelo período de 60 dias entre os meses de fevereiro e março de 2016 e os voluntários convidados recebiam uma comunicação eletrônica com um hiperlink para acesso ao questionário específico de seu perfil.

O número total de voluntários que contribuíram com a pesquisa foi de 147 , sendo 60 professores e 87 alunos, sendo que desses 16 responderam aos dois questionários por serem professores e também estarem matriculados em algum curso de formação.

Após o período estabelecido, foram gerados relatórios quantitativos para analisar as perguntas com respostas fechadas e planilhas de dados com as questões abertas para que as mesmas fossem agrupadas, classificadas e analisadas.

\section{ANÁLISE dOS RESULTADOS}

Com relação ao perfil dos docentes entrevistados observamos que mesmo atuando em diversos níveis educacionais a totalidade deles possuíam pelo menos um dispositivo móvel, e que possuíam alguma experiência com aplicações mobiles. Os alunos, também declaram em sua totalidade possuir pelo menos um dispositivo móvel e demostraram já possuir conhecimentos sobre o uso de aplicativos móveis. 
V Congresso Brasileiro de Informática na Educação (CBIE 2016)

Anais do XXVII Simpósio Brasileiro de Informática na Educação (SBIE 2016)

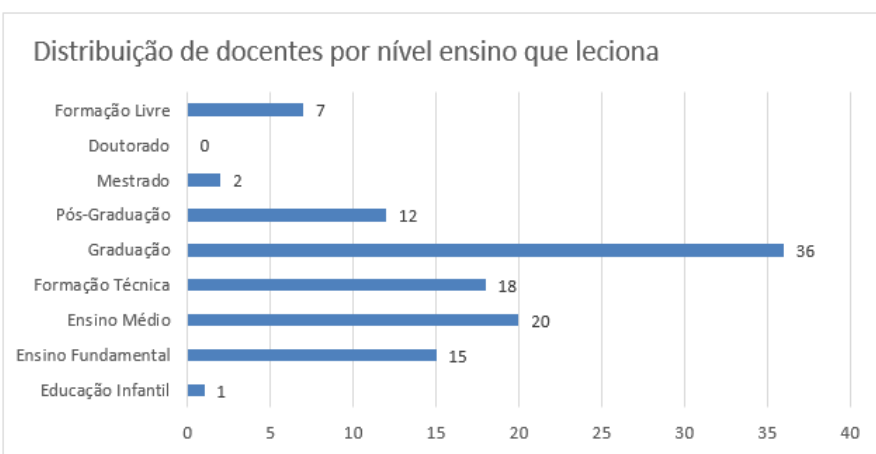

Gráfico 1 - Distribuição de docentes e discentes por nível de escolaridade

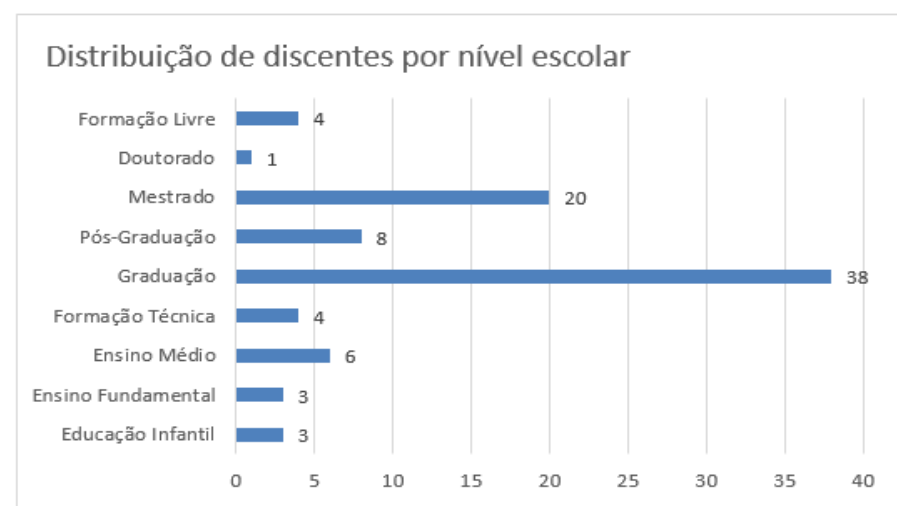

\section{Gráfico 2 - Distribuição de docentes e discentes por nível de escolaridade}

Inicialmente a pesquisa procurou identificar quais seriam os aplicativos de maior predileção dos alunos. O questionário apresentava uma lista com 07 tipos de aplicativos selecionados a partir do mapeamento sistemático de Cruz e Monteiro (2015) no qual o aluno poderia selecionar mais de um item. Ao final observamos as seguintes indicações:

Tabela 1- Preferencia de aplicativos dos alunos

\begin{tabular}{l|c} 
Tipo de aplicativo & Preferencia \\
\hline Jogos de perguntas e respostas sobre o assunto & 51 \\
\hline RPG & 49 \\
\hline Simuladores & 36 \\
\hline Consulta de vídeos sobre o assunto & 34 \\
\hline Consulta de textos sobre o assunto & 19 \\
\hline Outros & 7
\end{tabular}

Quanto a predileção dos alunos podemos observar uma heterogenia em relação aos aplicativos com uma pequena tendência a aplicações que usam jogos de perguntas e respostas, os chamados Quizz, com 26\% das indicações e jogos de RPG, com 25\% das indicações.

Como essa pergunta permitia que os alunos pudessem indicar mais de uma aplicação observamos uma similaridade entre as indicações.

Entre os alunos que indicaram que tinham predileção por jogos de perguntas e resposta, 38\% também indicaram jogos de RPG, 21\% indicaram Simuladores e 20\% indicaram Consulta de vídeos sobre o assunto.

Recomendamos que sempre que um professor ou pesquisador for iniciar um 
V Congresso Brasileiro de Informática na Educação (CBIE 2016)

Anais do XXVII Simpósio Brasileiro de Informática na Educação (SBIE 2016)

projeto para o desenvolvimento de OAM, procure conhecer as preferências dos alunos envolvidos, e faça um estudo para a adequação dos conteúdos para essa plataforma.

As plataformas mobiles são em geral muito diversificadas o que com certeza resulta em uma grande gama de OAMs que podem ser desenvolvidos, a pesquisa indicou que mesmo alguns tipos de aplicativos tendo uma maior aceitação que outros (Jogos de perguntas e respostas e RPG) a uma diversificação de preferencias e mesmo a possibilidade do desenvolvimento de aplicativos híbridos que permitam o uso de mais que um desses tipos de aplicação.

Em relação ao estilo de linguagem a ser adotada nas interfaces de um OAM, existe uma divergência entre professores e alunos, enquanto parte dos professores entrevistados (31 professores) preferem a adoção de uma linguagem mais formal, a maioria dos alunos se sente mais estimulados a utilizar softwares com interfaces que utilizem uma linguagem mais coloquial (49 alunos).

Tabela 2 - Preferencia de estilo de linguagem nas interfaces

\begin{tabular}{l|r|r} 
Estilo linguagem & Docentes & Discentes \\
\hline Coloquial & & \\
(Informal) & 22 & 49 \\
\hline Formal & 31 & 27 \\
\hline Técnica & 7 & 11 \\
\hline & &
\end{tabular}

Nos casos em que o OAM utilizar níveis de serviço diferenciados para professores e alunos ou interfaces que serão acessadas somente por um dos dois, o desenvolvedor deverá atentar-se em usar uma linguagem mais coloquial para alunos e mais formal para professores, sempre se atentando ao fato de que por ser um software educacional, mesmo interfaces que não exibam os conteúdos didáticos devem possuir uma grafia correta.

Os desenvolvimentos de OAM devem, segundo 100\% dos professores entrevistados, possuir ferramentas de feedback. Esta também é a opinião de $86 \%$ alunos. O ponto divergente é que para os alunos esse feedback deve ser síncrono enquanto para os professores, o OAM deve possuir ferramentas assíncronas como FAQs e mensagens enviadas por e-mail ou SMS.

Os desenvolvedores devem, sempre que possível, procurar criar ferramentas que possibilitem a adequação ao trinômio de interatividade abaixo demonstrado.

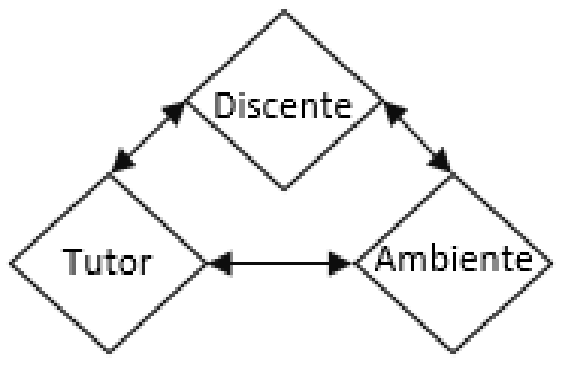

Figura 1 - Gráfico do trinômio de interatividade em OAM

Em relação a recursos multimídia o desenvolvedor deverá, sempre que possível, utilizar os diferentes recursos disponíveis dos dispositivos móveis para auxiliar os professores a transmitir os conteúdos didáticos. O OAM deve permitir que os professores 
V Congresso Brasileiro de Informática na Educação (CBIE 2016)

Anais do XXVII Simpósio Brasileiro de Informática na Educação (SBIE 2016)

possam disponibilizar recursos multimídia de forma simples, e sempre que possível, auxiliado com ferramentas tutoriais, conforme indicado por $75 \%$ dos professores entrevistados.

Tabela 3 - Recursos multimídias que os discentes mais gostam de usar em OAMs

\begin{tabular}{|l|r|l|r|}
\hline Tipo de recurso & Gostam de usar & Recurso & Gostam de usar \\
\hline Textos objetivos & 44 & Imagens sequencias & 25 \\
\hline Vídeos & 44 & Hipertextos & 20 \\
\hline Áudios & 28 & Imagens estáticas & 17 \\
\hline
\end{tabular}

Ao realizarmos uma busca sistêmica três sistemas para dispositivos móveis mais utilizados pelos discentes, é possível encontrar diversas ferramentas nativas que podem auxiliar as pessoas com necessidades especiais, mas que infelizmente não são utilizadas pelos desenvolvedores. Ao avaliarmos a importância das ferramentas de acessibilidade 98\% indicaram que esse tipo de ferramenta deve estar presente em todos os OAM.

Um outro fator importante que foi percebido durante a pesquisa é que um percentual de professores entrevistados (11\%) aponta uma maior preferência ao uso de aplicações WEB em relação ao uso de aplicativos mobile, alegando dentre outros fatores o de que as interfaces dos aplicativos mobile, não são ideais para inserção de textos de maior tamanho e edições mais complexas, sendo este um dos critérios de dificuldade.

Mesmo o percentual não sendo muito grande, ele surgiu a partir de manifestações espontâneas em perguntas abertas do questionário. Cruz, Ramos e Rodrigues (2015) já recomendavam aos desenvolvedores a necessidade avaliar a usabilidade das interfaces das OAMs durante o projeto. Logo analisamos possíveis recomendações para os desenvolvedores de OAMs.

Quando for possível determinar que o tipo do dispositivo móvel será utilizado pela OAM, deve-se realizar testes de usabilidade de interfaces PUI (Pen-Based Interface) com usuários diferentes. Quando não houver a possibilidade de determinar o dispositivo móvel que será utilizado, o aplicativo deve ser construído utilizando técnicas de design responsivo, e sempre que possível as interfaces destinadas aos professores devem ser projetadas para serem usadas não apenas em dispositivos móveis, mas também em navegadores WEB.

Um outro fator impactante para o desenvolvimento de OAMs que devem ser considerados pelos desenvolvedores está ligado a qual linguagem de programação deve ser utilizada.

Uma das principais dificuldades que o desenvolvedor deve considerar no momento de escolher qual a linguagem de programação que deve ser utilizada no desenvolvimento de um OAM e que a mesma será utilizada por diferentes professores e alunos e que cada um deles tem o poder de escolha sobre o hardware e a plataforma a que desejam utilizar. Diferente de um projeto de $e$-learning no qual o foco do desenvolvedor está em um design agradável e suas dimensões eram limitadas por um navegador o projeto do desenvolvimento de um OAMs deve procurar soluções para as múltiplas plataformas existentes.

Ao levantarmos o perfil de professores e alunos, procuramos identificar quantos dispositivos móveis cada um deles possuía e quais sistemas operacionais eram utilizados nesses dispositivos. 
V Congresso Brasileiro de Informática na Educação (CBIE 2016)

Anais do XXVII Simpósio Brasileiro de Informática na Educação (SBIE 2016)

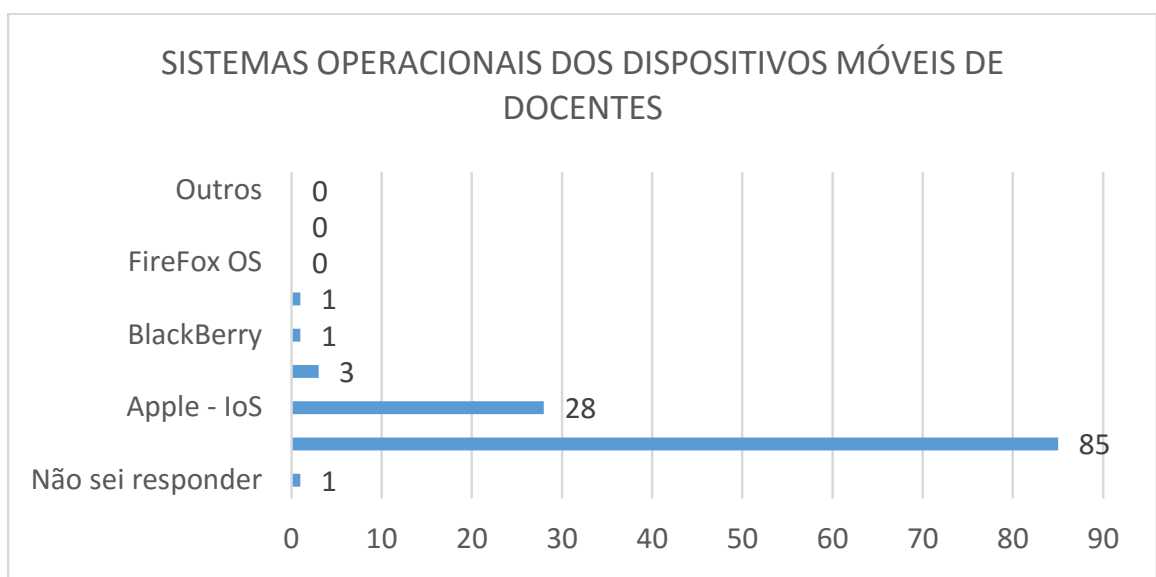

Gráfico 3 - Sistemas Operacionais dos Dispositivos Móveis dos professores entrevistados

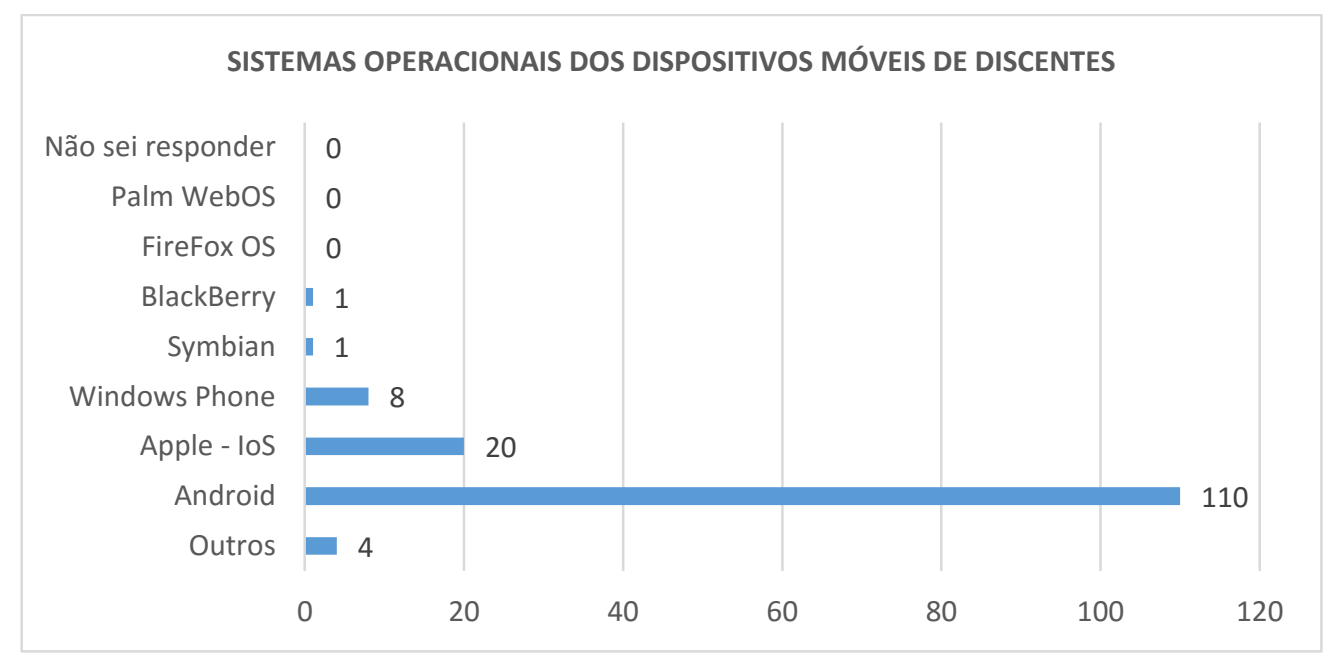

Gráfico 4 - Sistemas Operacionais dos Dispositivos Móveis dos alunos entrevistados

Ao totalizarmos os gráficos podemos observar que $74 \%$ dos entrevistados possuíam dispositivos móveis com sistema operacional Android, 18\% possuíam dispositivos móveis com sistema operacional IoS, 4\% possuíam dispositivos móveis com sistema operacional Windows Phone e mais $4 \%$ dos entrevistados possuíam dispositivos móveis com outros sistemas operacionais.

Com base na distribuição observada na pesquisa, consultamos as documentações disponibilizadas pelas empresas proprietárias dos três sistemas operacionais com maior percentual de usuários na pesquisa e obtivemos indicações para o uso das linguagens de programação abaixo relacionadas. A partir dessas recomendações analisamos a compatibilidade de cada uma das linguagens com os três sistemas operacionais.

Tabela 4 - Tabela de Compatibilidade de linguagem de programação para dispositivos móveis

\begin{tabular}{|l|l|c|c|c|}
\hline Linguagem & Fabricante & \multicolumn{3}{|c|}{ Compatibilidade } \\
\hline & & Android & loS & Windows Phone \\
\hline Swift & Apple & & X & \\
\hline Dart & Google & X & & X \\
\hline XAML & Microsoft & & & $X$ \\
\hline C\# & Microsoft & & & \\
\hline
\end{tabular}


V Congresso Brasileiro de Informática na Educação (CBIE 2016)

Anais do XXVII Simpósio Brasileiro de Informática na Educação (SBIE 2016)

\begin{tabular}{|l|l|c|c|c|}
\hline VisualBasic & Microsoft & & & $\mathrm{X}$ \\
\hline Java & Sun Microsystems & $\mathrm{X}$ & & $\mathrm{X}$ \\
\hline HTML5 & W3C & $\mathrm{X}$ & $\mathrm{X}$ & $\mathrm{X}$ \\
\hline
\end{tabular}

Fontes: HTML5 Mobile, Microsoft Brasil, Dart.org e Apple

Observamos que o percentual de entrevistados que possuem dispositivos com sistema operacional Android devem ser considerados no momento da escolha de uma linguagem e sempre que o projeto permitir que a instituição de ensino defina uma plataforma com um sistema operacional único esse sistema deverá ser considerado por oferecer uma maior familiaridade de uso para professores e alunos.

Quando não for possível determinar em qual plataforma o OAM será utilizado por professores e alunos deve-se prioritariamente utilizar como linguagem de desenvolvimento o HTML5 (HyperText Markup Language). O HTML5 permite o uso de outras linguagens, ferramentas e bibliotecas em complemento de suas funcionalidades, portanto o desenvolvedor deverá também avaliar o uso de cada uma delas procurando utilizar apenas as que apresentarem compatibilidade com os sistemas operacionais utilizados por professores e alunos, conseguindo com isto minimizar esforços de desenvolvimento do OAMs e consequentemente os custos envolvidos.

Ao considerar a análise dos dados obtidos nesse estudo os desenvolvedores deverão, sempre que possível, buscar alinhar seus projetos as recomendações aqui indicadas, facilitar o uso de ferramentas de acessibilidade, desenvolver aplicações do design responsivo, complementos de compatibilidade com leitores de texto e vídeo descrição e interfaces com opção de configuração de cores e outros recursos que devem sempre estar disponível para professores e alunos.

\section{CONSIDERAÇÕES FINAIS}

Neste artigo foi realizado um estudo sobre a opinião de professores e alunos sobre os requisitos funcionais e didáticos, e sobre tópicos como linguagem, interatividade, recursos multimídias e acessibilidade em relação aos OAMs, visando auxiliar pesquisadores e desenvolvedores quanto aos requisitos para o desenvolvimento de ferramentas mais atrativas para o mobile learning.

Inicialmente investigamos as preferências de alunos sobre os tipos de OAMs e observamos que não existe uma tendência a um único tipo de OAM. Desenvolvedores, pesquisadores e professores devem adaptar seus conteúdos ao mobile learning usando para isso um ou mais tipos de OAM.

Observamos, também, que professores e alunos concordam em pontos importantes como a presença de opções de configurações para pessoas com necessidades especiais e a necessidade dos OAMs promoverem uma maior interatividade entre professores e alunos. Também observamos pontos de discordância entre os grupos entrevistados tais como a melhor linguagem a ser usada nas interfaces da OAM e no uso de ferramentas de interatividade síncronas, ou assíncronas para feedbacks. Tais opiniões devem ser usadas como uma orientação para desenvolvedores e pesquisadores na criação de novas OAMs.

Um outro ponto importante a ser considerado pelos desenvolvedores de OAMs é a linguagem de programação a ser observada, pois em muitos casos determinar uma 
V Congresso Brasileiro de Informática na Educação (CBIE 2016)

Anais do XXVII Simpósio Brasileiro de Informática na Educação (SBIE 2016)

única plataforma para o uso da OAM poderá torná-la inacessível para alunos que usem outras plataformas. Como auxílio aos desenvolvedores indicamos que antes de iniciar o desenvolvimento de um OAM, o mesmo analise a possibilidade de usar linguagem multiplataforma.

Após a realização do estudo aqui apresentado podemos sugerir aos desenvolvedores que ao iniciarem um projeto para o desenvolvimento de um OAM avaliem a possibilidade do uso de ferramentas que estimulem os alunos a buscar o aprimoramento do conhecimento por conta própria em qualquer ambiente, que considere a designer e outros recursos para estimular alunos de faixas etárias e condições físicas diferenciadas (investindo em opções de acessibilidade) e estimulado, sempre que possível, a comunicação entre aluno, professor e ambiente de estudo.

Recomendamos, também, que o desenvolvedor considere linguagens de desenvolvimento que atendam a diversas plataformas, como o HTML5 por exemplo, layouts responsos que atendam aos diversos formatos de dispositivos móveis e quando não for possível determinar quais dispositivos móveis serão utilizados o desenvolvedor deverá usar como referência para a escolha de requisitos soluções que atendam a maioria dos alunos e professores que irão utilizar o OAM.

Como contribuições futuras, iremos desenvolver, a partir das definições de requisitos dessa pesquisa, um protótipo de um OAM e submete-lo novamente a avaliação de professores e alunos visando reiterar os requisitos investigados nesse estudo.

\section{REFERENCIAS}

Cruz, J.R.U., \& Monteiro A. M. (2015). Mapeamento Sistemático sobre experimentos educacionais com uso de dispositivos móveis, Anais XI Workshop Computação da FACCAMP, pp.91.

Cruz, J.R.U., Ramos, R., Rodrigues, \& W.B. (2015). Guideline para desenvolvimento de aplicativos mobile, Anais XI Workshop Computação da FACCAMP, pp.75.

Moreira, M. B., \& Conforto, D. (2011). Objetos de Aprendizagem: Discutindo a Acessibilidade e a Usabilidade. XXII SBIE - XVII WIE - SBC, pp. 390-393.

Sharp, H., Taylor, J., Löber, A., Frohberg, D., Mwanz, D., \& Murelli, E. (2003). Establishing user requirements for a mobile learning environment.

Yen, J. C., \& Lee, C. Y. (2011). Exploring problem solving patterns and their impact on learning achievement in a blended learning environment. Computers \& Education, 56(1), 138-145.

Weiser, M. (1993). Some computer science issues in ubiquitous computing. Communications of the ACM, 36(7), 74-83. In Special Issue, Computer-Augmented Environments [verified 1 Oct 2004]

Georgieva E. (2007). A Comparison Analysis of Mobile Learning Systems, In: Communication and Cognition Journal, Vol 40, Numb 3/4, Belgium. 